\title{
Persistência de Sulfentrazone em Argissolo Vermelho-Amarelo Cultivado COM CANA-DE-AçÚCAR ${ }^{1}$
}

\author{
Persistence and Residual Effect of Sulfentrazone on Typic Hapludalf with Sugar-Cane
}

\author{
VIVIAN, R. ${ }^{2}$, REIS, M.R. ${ }^{3}$, JAKELAITIS, A. ${ }^{4}$, SILVA, A.F. ${ }^{3}$, GUIMARÃES, A.A. ${ }^{5}$, SANTOS, J.B. ${ }^{6}$ e \\ SILVA, A.A. ${ }^{7}$
}

\begin{abstract}
RESUMO - Avaliou-se neste trabalho a persistência do sulfentrazone em Argissolo VermelhoAmarelo (PVA) e seu efeito na microbiota do solo cultivado com cana-de-açúcar. Os tratamentos constituíram-se de aplicações do herbicida somente no ano de 2003, em 2003 com reaplicação em 2004 e da testemunha sem o herbicida. Nas subparcelas e subsubparcelas foram estudadas a distribuição do herbicida no perfil do solo (0-10 e $10-20 \mathrm{~cm}$ de profundidade) e a sua persistência aos 467/24, 517/74, 550/107 e 640/197 dias após aplicação (DAA), em 2003/2004, respectivamente. A quantificação indireta dos resíduos foi realizada por bioensaio, e a evolução de $\mathrm{C}-\mathrm{CO}_{2}$, juntamente com a determinação da biomassa microbiana (CBM), foram avaliadas em solo coletado aos 640/197 DAA. Constatou-se redução de massa seca da parte aérea de Sorghum vulgare durante todo o período avaliado, quando o herbicida foi aplicado em 2003 e reaplicado em 2004. A maioria dos resíduos de sulfentrazone foi detectada na profundidade de $0-10 \mathrm{~cm}$, sendo pouco significativo seu potencial de lixiviação no solo nas condições ambientais em que foi conduzido o experimento. $\mathrm{O}$ sulfentrazone influenciou a evolução de $\mathrm{C}-\mathrm{CO}_{2}$ e CBM do solo, observando-se o maior valor acumulado de $\mathrm{C}-\mathrm{CO}_{2}$ no tratamento sem a aplicação do herbicida. O sulfentrazone apresentou elevada persistência no PVA, com efeito negativo sobre microrganismos do solo, porém sem representar riscos de lixiviação no seu perfil.
\end{abstract}

Palavras-chave: herbicida, resíduos, bioensaio, (Saccharum spp.), atividade microbiana.

\begin{abstract}
The objective of this study was to evaluate sulfentrazone persistence in Typic Hapludalf and its effect on the microbiota of soil cultivated with sugar-cane. The treatments consisted of herbicide application only in 2003, in 2003 with reapplication in 2004 and control without herbicide. Herbicide distribution in the soil profile $(0-10$ and $10-20 \mathrm{~cm}$ of depth) and persistence in 467/24, 517/74, 550/107 and 640/197 days after application (DAA), were studied in the split-plots and split-split-plots, respectively. Indirect quantification of the residues was accomplished by bioassay and $\mathrm{C}-\mathrm{CO}_{2}$ evolution together with microbial biomass (CBM) determination were evaluated in soil collected 640/197 DAA. Reduction of dry mass of the aerial part of Sorghum vulgare was verified during the entire evaluated period when the herbicide was applied in 2003 and reapplied in 2004. Most of the residues were detected at the depth of $0-10 \mathrm{~cm}$, with their leaching potential in the soil being of little significance under environmental conditions. Sulfentrazone influenced the evolution of $\mathrm{C}_{-} \mathrm{CO}_{2}$ and $\mathrm{CBM}$ of the soil, with the largest accumulated value of $\mathrm{C}_{-} \mathrm{CO}_{2}$ being observed for the treatment without herbicide application. Sulfentrazone presented high persistence in PVA, with a negative effect on soil microorganisms, but without posing leaching risks in its profile.
\end{abstract}

Keywords: herbicide, residues, bioassay, (Saccharum spp.), microbial activity.

Recebido para publicação em 21.3.2006 e na forma revisada em 10.11.2006.

Doutorando em Fitotecnia, Departamento de Fitotecnia, Escola Superior de Agricultura "Luiz de Queiroz" - ESALQ/USP, Av. Pádua Dias, 11 Caixa Postal 9, 13418-900 Piracicaba-SP, <agrovivian@yahoo.com.br>; ${ }^{3}$ Mestrando em Fitotecnia, Departamento de Fitotecnia,Universidade Federal de Viçosa - DFT/UFV. ${ }^{4}$ Professor, Fundação Universidade Federal de Rondônia - UNIR. ${ }^{5}$ Professor, Universidade Vale do Rio Doce - UNIVALE. ${ }^{6}$ Graduanda em Agronomia - DFT/UFV; ${ }^{7}$ Professor Adjunto DFT/UFV. 


\section{INTRODUÇÃO}

O uso de defensivos agrícolas tem proporcionado, juntamente com os demais avanços tecnológicos, incrementos produtivos e redução nas perdas ocasionadas por pragas, doenças e plantas daninhas. Todavia, a utilização desses compostos de modo indiscriminado representa alto risco de contaminação ambiental.

Os herbicidas, ao serem aplicados em pré ou pós-emergência, acabam direta ou indiretamente alcançando o solo (Law, 2001), podendo causar danos às culturas subseqüentes (Silva et al., 1999; Gonçalves et al., 2001), à flora e à fauna do solo, além da contaminação de águas superficiais e subterrâneas. Segundo Filizola et al. (2002), ao alcançarem o solo, dá-se início ao processo de redistribuição e degradação desses compostos, o qual pode ser extremamente curto, como o que ocorre com algumas moléculas simples e não-persistentes, ou perdurar por meses ou anos, tal como ocorre com compostos altamente persistentes.

Em condições normais, a quantidade do herbicida perdido pela movimentação no perfil do solo está, geralmente, entre 0,1 e 1\% do total aplicado, mas, em determinadas circunstâncias, esse percentual pode ser igual ou superior a 5\% (Carter, 2000). Entretanto, o potencial de contaminação do lençol freático por herbicidas ou a sua permanência nas camadas superficiais do solo dependem, principalmente, de sua mobilidade no perfil do solo, a qual está direta e inversamente vinculada à sua capacidade de adsorção e degradação. Para Hinz (2001), a capacidade de sorção de herbicidas e sua degradação no solo por processos físicos, químicos e biológicos são fundamentais para determinar se o produto usado terá ou não efeito na qualidade ambiental.

O material de origem do solo, os tipos de minerais predominantes na fração argila, os grupos funcionais e a quantidade da matéria orgânica, além das condições climáticas do local, são responsáveis pelas variações de adsorção e comportamento de herbicidas nos solos (Carter, 2000; Weber et al., 2004). Para uso seguro de herbicidas, além do conhecimento dessas características, é necessário conhecer a duração de sua atividade, o processo de controle de sua persistência, a sua seletividade entre as culturas e plantas daninhas e os possíveis efeitos secundários sobre microrganismos do solo (Blanco, 1979). Avaliações mais amplas sobre o efeito de herbicidas no ambiente, as quais incluem a incorporação de indicadores microbiológicos, como a respiração basal (evolução do $\mathrm{C}-\mathrm{CO}_{2}$ ), a biomassa microbiana dos solos e o quociente metabólico, têm permitido antecipar a constatação de alterações na dinâmica dos ecossistemas (Anderson \& Domsch, 1993; Valpassos et al., 2001; Santos et al., 2005). O quociente metabólico possibilita que se avalie, também, o estado de equilíbrio no ambiente do solo; quanto menores os valores observados, mais próximo este se encontra da estabilidade (Tótola \& Chaer, 2002).

O herbicida sulfentrazone, pertencente ao grupo das aril-triazolinonas, é inibidor da protoporfirinogênio oxidase (PROTOX), a qual é responsável pela oxidação do protoporfirinogênio a protoporfirina IX, na biossíntese da clorofila. Esse herbicida possui excelente atividade pré-emergente no solo para controle de plantas daninhas dicotiledôneas e diversas espécies monocotiledôneas (FMC corp., 1995), sendo amplamente utilizado no controle de plantas daninhas em cana-de-açúcar e também de biótipos resistentes à acetolactato sintase - ALS, na cultura da soja (Hulting et al., 2001). Possui registro para culturas de grande importância comercial para o Brasil, como a cana-de-açúcar, a soja e o eucalipto. A sua meia-vida no solo $\left(\mathrm{t}_{1 / 2}\right)$ é estimada entre $110 \mathrm{e}$ 280 dias, variando a partir das condições edafoclimáticas locais, sendo a atividade microbiológica seu mecanismo inicial de degradação (FMC corp., 1995). Além disso, possui baixa dissociação em água, comportando-se como ácido fraco ( $\mathrm{pKa}=6,56)$, e sua solubilidade aumenta com o aumento do $\mathrm{pH}(\mathrm{pH} 6,0=$ $110 \mathrm{mg} \mathrm{L}^{-1}$; pH 7,5 = $1.600 \mathrm{mg} \mathrm{L}^{-1}$ ), encontrando-se predominantemente na solução do solo na forma não-ionizada (FMC corp., 1995).

Considerando a longa persistência do sulfentrazone (Grey et al., 2000; Polubesova et al., 2003) e os problemas ocasionados pelo seu efeito carryover em rotação de culturas (Main et al., 2004), avaliou-se neste trabalho a persistência e o efeito desse herbicida na microbiota do solo, em Argissolo VermelhoAmarelo (PVA) - MG cultivado com cana-deaçúcar, visando definir técnicas para o uso seguro de sulfentrazone. 


\section{MATERIAL E MÉTODOS}

O experimento foi realizado sobre Argissolo Vermelho-Amarelo (PVA) cultivado com canade-açúcar, no município de Ponte Nova-MG, situado a $20^{\circ} 20^{\prime} \mathrm{S}$ e $43^{\circ} 48^{\prime} \mathrm{W}$. O cultivo de cana-planta foi iniciado em março de 2003, com continuação de cana-soca até maio de 2005, utilizando-se o cultivar SP 801816. A caracterização física e química do solo (Tabela 1) foi realizada utilizando-se amostras coletadas em duas profundidades (0-10 e $10-20 \mathrm{~cm})$. A adubação utilizada foi de $500 \mathrm{~kg}$ da formulação NPK 8-28-16, juntamente ao plantio, com adubação suplementar em cobertura em 2004.

O delineamento experimental utilizado foi de blocos completos ao acaso, com três repetições e arranjo em parcelas subsubdivididas, sendo as parcelas (5,6 x $5 \mathrm{~m})$ compostas pela aplicação do herbicida em 2003, aplicação em 2003 com reaplicação no ano de 2004 e testemunha sem sulfentrazone. As subparcelas e subsubparcelas constituíram-se das profundidades (0-10 e 10-20 cm) e épocas de coleta do solo, respectivamente. As amostragens do solo foram realizadas em quatro épocas: 467/ $24,517 / 74,550 / 107$ e 640/197 dias após a aplicação do herbicida (DAA), em 2003/2004, respectivamente.

As pulverizações de sulfentrazone foram realizadas em pré-emergência, em condições de solo úmido, na dose de $900 \mathrm{~g} \mathrm{ha}^{-1}$, aplicando-se o equivalente a $200 \mathrm{~L} \mathrm{ha}^{-1}$. Os dados referentes a precipitação pluvial, temperatura e umidade relativa do ar durante o período experimental encontram-se na Figura 1.
Para avaliação da persistência do sulfentrazone no solo, utilizou-se o bioensaio como método indireto de quantificação dos resíduos (Szmigielska et al., 1998). Para isso, em ensaio preliminar, selecionaram-se espécies bioindicadoras para o herbicida, adotando-se o sorgo (Sorghum vulgare), cv. Esmeralda, como espécie adequada aos ensaios. Posteriormente, utilizando-se dois substratos - areia lavada e o solo em estudo (PVA) - isentos de herbicidas, determinaram-se em casa de vegetação as curvas de dose-resposta. Para isso, soluções com as doses crescentes de sulfentrazone 10 ; 1,$95 ; 3,9 ; 7,81 ; 15,62 ; 31,25 ; 62,50 ; 125,0 ;$ e 250,0 $\mathrm{g} \mathrm{ha}^{-1}$ ) foram aplicadas em pré-emergência sobre vasos contendo 120 g dos substratos,

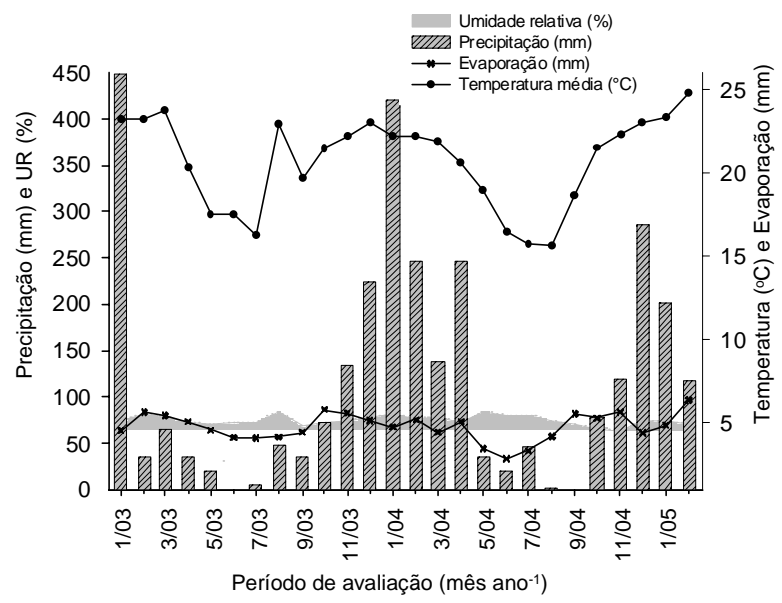

Figura 1 - Médias mensais de umidade relativa (\%), precipitação pluvial $(\mathrm{mm})$, evaporação $(\mathrm{mm})$ e temperatura média do ar $\left({ }^{\circ} \mathrm{C}\right)$ observadas na estação experimental de Ponte NovaMG $\left(20^{\circ} 20^{\prime} \mathrm{S}\right.$ e $\left.43^{\circ} 48^{\prime} \mathrm{W}\right)$ durante a condução do experimento, entre 2003 e 2005.

Tabela 1 - Principais características físicas e químicas do Argissolo Vermelho-Amarelo - PVA, cultivado com cana-deaçúcar, no período compreendido entre março/2003 e maio/2005, conforme a sua profundidade de amostragem. ViçosaMG, 2005

\begin{tabular}{|c|c|c|c|c|c|c|c|c|c|c|}
\hline \multirow{2}{*}{$\begin{array}{l}\text { Profundidade } \\
\text { do solo } \\
(\mathrm{cm})\end{array}$} & \multirow[t]{2}{*}{$\mathrm{pH}$} & $\mathrm{H}^{+}+\mathrm{Al}^{+3}$ & $\mathrm{SB}$ & $\begin{array}{c}\text { CTC } \\
\text { (t) }\end{array}$ & $\begin{array}{c}\text { CTC } \\
(\mathrm{T})\end{array}$ & MO & $\mathrm{V}$ & Argila & Areia & \multirow[t]{2}{*}{ Classe textural } \\
\hline & & \multicolumn{4}{|c|}{$\mathrm{cmol}_{\mathrm{c}} \mathrm{dm}^{-3}$} & \multicolumn{4}{|c|}{$(\%)$} & \\
\hline $0-10$ & 6,6 & 1,32 & 3,19 & 3,19 & 4,51 & 1,19 & 71 & 29 & 43 & \multirow{2}{*}{ Franco-Argilo-Arenosa } \\
\hline $10-20$ & 6,8 & 1,32 & 3,05 & 3,05 & 4,37 & 0,79 & 70 & 28 & 47 & \\
\hline
\end{tabular}

pH em água: relação 1:2,5. $\mathrm{H}+\mathrm{Al}^{+3}$ : extrator acetato de cálcio $0,5 \mathrm{~mol} \mathrm{~L}^{-1}$, a pH 7,0. CTC (T) - pH 7,0. 
nos quais se semearam cinco sementes de S. vulgare. Aos 21 dias após aplicação do herbicida, avaliaram-se a fitotoxicidade (escala EWRC, 1964), a altura e a massa seca da parte aérea das plantas. Para interpretação dos resultados, os valores foram comparados ao tratamento sem herbicida (dose zero), sendo submetidos à análise estatística, utilizando-se o modelo loglogístico não-linear $\hat{Y} \quad b \square\left\{a \square b /\left(1 \square\left(x / I_{50}\right)^{c}\right\}\right.$ (Seefeldt et al., 1995; Stork \& Hannah, 1995), em que $a$ e $b$ correspondem ao nível máximo e mínimo da curva de dose-resposta; $c$, ao declive da curva em torno do $\mathrm{I}_{50} ; X$, às doses do herbicida avaliadas; e $\mathrm{I}_{50}$, à dose-resposta referente à redução de 50\% da massa seca da parte aérea da planta indicadora. Os valores obtidos da $\mathrm{I}_{50}$ em solo e areia foram utilizados para cálculo da relação adsortiva (RA) do herbicida no solo, expresso pela fórmula RA = $\left[\left(\mathrm{I}_{50}\right.\right.$ solo $-\mathrm{I}_{50}$ areia $) / \mathrm{I}_{50}$ areia $]$.

Posteriormente, nas suas respectivas épocas de coleta, o solo PVA foi amostrado em campo (amostra homogeneizada composta por duas frações em cada parcela), nas profundidades de 0 a 10 e 10 a 20 cm, secado à sombra e peneirado em malha de $2 \mathrm{~mm}$, sendo submetido ao mesmo procedimento de avaliação usado para confecção da curva de dose-resposta, porém sem a aplicação do herbicida. Para cada amostra coletada em campo, foram utilizadas quatro repetições em casa de vegetação, totalizando, ao final do experimento, 288 vasos. Os valores obtidos de fitotoxicidade, altura e massa seca da parte aérea das plantas foram comparados aos do solo coletado na parcela testemunha (sem aplicação do herbicida); posteriormente, para quantificação dos resíduos deste no solo, utilizou-se a curva de dose-resposta previamente determinada (Figura 3).

Avaliaram-se, também, na amostragem efetuada aos 640/197 DAA, a evolução de C- $\mathrm{CO}_{2}$ e o carbono da biomassa microbiana (CBM) do solo $(0-10 \mathrm{~cm})$. Para isso, utilizou-se o método respirométrico de avaliação do $\mathrm{C}-\mathrm{CO}_{2}$, no qual amostras de 100,00 g de solo úmido (80\% da capacidade de campo) e peneirado foram incubadas durante 28 dias em frascos de erlenmeyer hermeticamente fechados. $\mathrm{O} \mathrm{C}-\mathrm{CO}_{2}$ liberado do solo foi carreado por fluxo contínuo de ar (isento de $\mathrm{CO}_{2}$ ) até outro

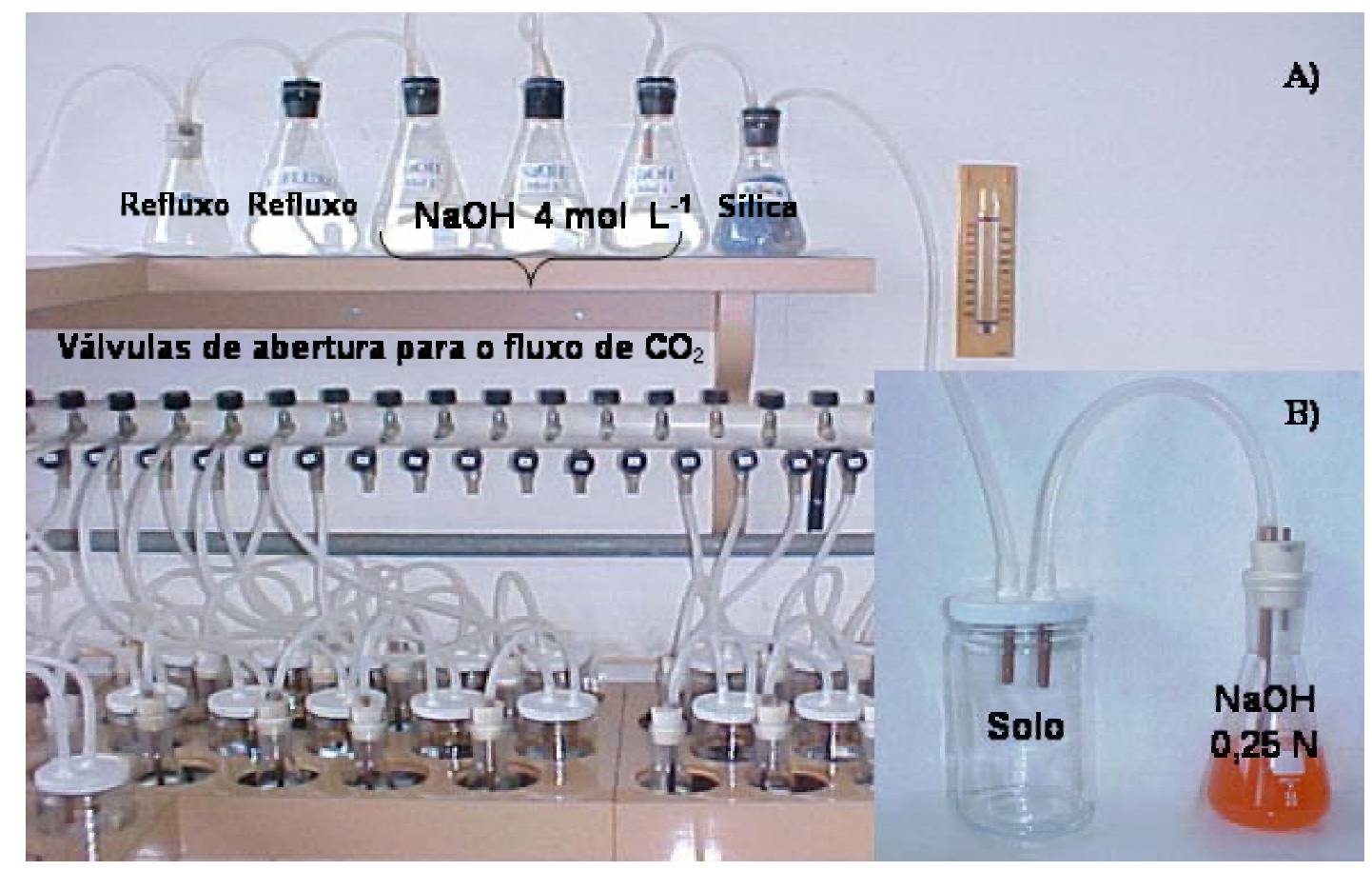

Figura 2 - A) - equipamento respirométrico utilizado para avaliação da evolução de C- $\mathrm{CO}_{2}$, em Argissolo Vermelho-Amarelo incubado durante 28 dias a $80 \%$ da sua capacidade de campo; e B) - unidade experimental de coleta do $\mathrm{C}_{-} \mathrm{CO}_{2}$ evoluído. 


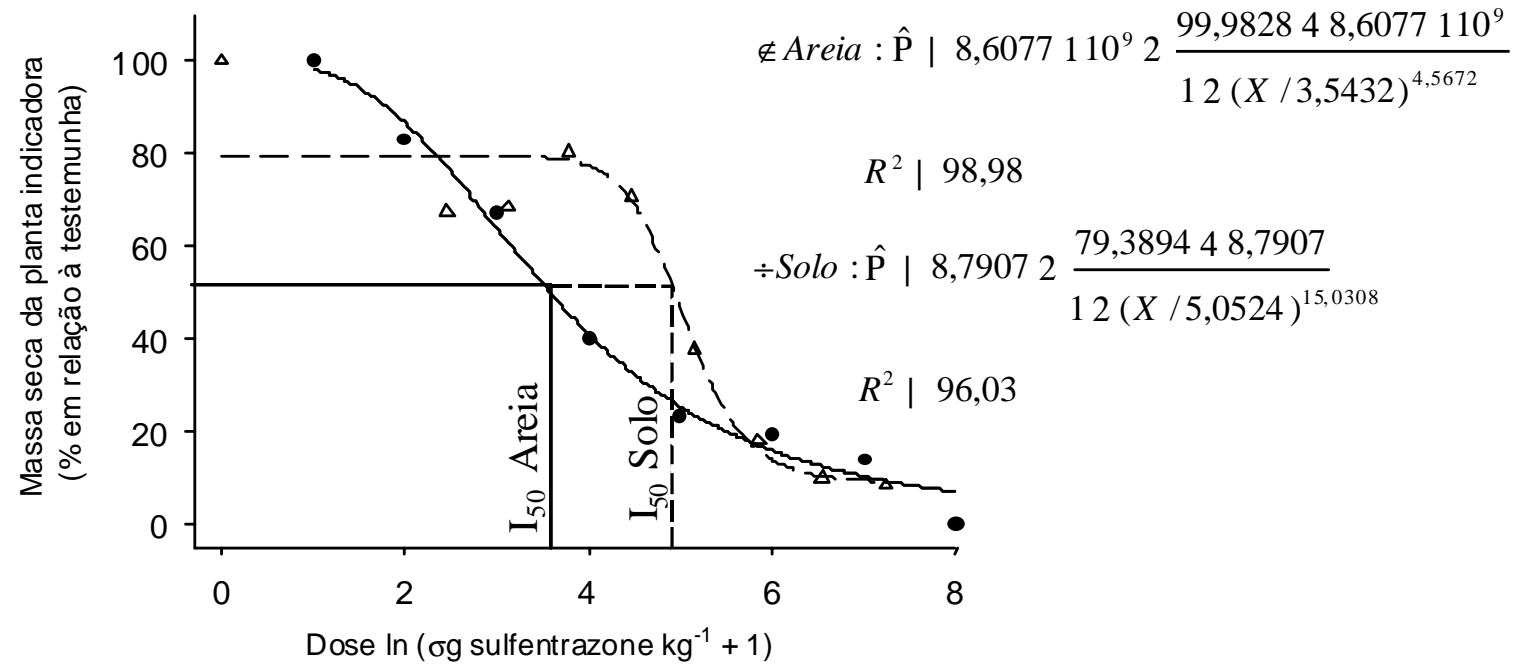

Figura 3 - Curva de dose-resposta obtida para massa seca da parte aérea (\% em relação à testemunha) de Sorghum vulgare, avaliada aos 21 dias após aplicação do herbicida, em função de doses crescentes de sulfentrazone $(X)$ aplicadas em Argissolo VermelhoAmarelo (-- ) e em areia (-).

frasco contendo $100 \mathrm{~mL}$ de solução de $\mathrm{NaOH}$ $0,25 \mathrm{~mol} \mathrm{~L}^{-1}$ (Figura 2). A cada sete dias, avaliou-se a quantidade de $\mathrm{C}-\mathrm{CO}_{2}$ evoluído a partir da titulação de $10,00 \mathrm{~mL}$ da solução de $\mathrm{NaOH}$ com solução de $\mathrm{HCl} 0,1 \mathrm{~mol} \mathrm{~L}{ }^{-1}$, previamente padronizado, preenchendo-se novamente os erlenmeyers com $100 \mathrm{~mL}$ de solução de $\mathrm{NaOH}$ 0,25 $\mathrm{mol} \mathrm{L}^{-1}$. Utilizou-se, como alternativa de controle da qualidade do ar carreado, frasco de erlenmeyer sem solo, que serviu como amostra "branco" em relação às demais. A temperatura da sala foi monitorada, permanecendo entre 24 e $27^{\circ} \mathrm{C}$.

Ao término do período de incubação, o solo foi retirado dos erlenmeyers, tomando-se $20 \mathrm{~g}$ de cada frasco para determinação do carbono da biomassa microbiana (CBM). Utilizou-se o método descrito por Vance et al. (1987) modificado por Islam \& Weil (1998), no qual as amostras foram tratadas com radiação de microondas por tempo previamente calculado $(60+60$ seg), em vez da fumigação com clorofórmio. O CBM foi extraído das amostras (irradiadas e não-irradiadas) de solo com $80 \mathrm{~mL}$ da solução de $\mathrm{K}_{2} \mathrm{SO}_{4} 0,5 \mathrm{~mol} \mathrm{~L}^{-1}$. Após a adição da solução de $\mathrm{K}_{2} \mathrm{SO}_{4}$, as amostras foram submetidas a agitação, por 30 minutos, em mesa agitadora horizontal, permanecendo, em seguida, em repouso durante mais 30 minutos até a filtragem em filtros de papel Whatman no 42. Em tubo digestor, tomaram-se $10 \mathrm{~mL}$ do filtrado, ao qual foram adicionados os reagentes, na seguinte ordem: 2,0 mL de solução de $\mathrm{K}_{2} \mathrm{Cr}_{2} \mathrm{O}_{7} 0,0667 \mathrm{~mol} \mathrm{~L}^{-1}$, $5 \mathrm{~mL}$ de $\mathrm{H}_{3} \mathrm{PO}_{4}$ concentrado e $10,0 \mathrm{~mL}$ de solução de $\mathrm{H}_{2} \mathrm{SO}_{4} 0,2 \mathrm{~mol} \mathrm{~L}^{-1}$. Posteriormente, os tubos foram aquecidos por $30 \mathrm{~min}$ a $100^{\circ} \mathrm{C}$, deixando-se esfriar até atingir a temperatura ambiente $\left(24-26^{\circ} \mathrm{C}\right)$. O volume foi completado para $100,0 \mathrm{~mL}$ em proveta calibrada. Transferiu-se a amostra para frascos erlenmeyers de $250 \mathrm{~mL}$, adicionou-se o indicador difenilamina (5 gotas) e procedeu-se à titulação com solução $0,033 \mathrm{~mol} \mathrm{~L}^{-1}$ de $\left(\mathrm{NH}_{4}\right)_{2} \mathrm{Fe}\left(\mathrm{SO}_{4}\right)_{2}$, até mudança da cor azul-escura para verde. A partir dos valores obtidos da evolução do C-CO2 e CBM, calculou-se o $\mathrm{qCO}_{2}$ ( $\mathrm{mg} \mathrm{mg}^{-1} \mathrm{dia}^{-1}$ de C- $\mathrm{CO}_{2}$ ), dividindo-se a média diária do $\mathrm{C}-\mathrm{CO}_{2}$ obtida a partir do valor total aos 28 dias de incubação pelo CBM determinado no solo.

Os resultados foram submetidos à análise da variância e, quando significativos, realizaram-se comparações de médias pelo teste de Tukey a $5 \%$ de probabilidade. Para avaliação dos indicadores microbiológicos (evolução do C- $\mathrm{CO}_{2}$, CBM e $\mathrm{QM}$ ), os dados foram submetidos à análise de agrupamento hierárquico dos tratamentos, com base no método de ligação média e na distância euclidiana. Posteriormente, após a formação dos grupos, foi avaliado o percentual de acertos pela análise discriminante. 


\section{RESULTADOS E DISCUSSÃO}

O modelo log-logístico utilizado ajustouse satisfatoriamente às observações de massa seca da parte aérea de $S$. vulgare cultivado em solo PVA e areia lavada, satisfazendo a premissa de aceite do modelo com base no seu significado biológico (Figura 3). Os valores obtidos para $\mathrm{I}_{50}$ de 3,5432 em areia e de $5,0524 \mathrm{Pg} \mathrm{kg}^{-1}$ em solo, correspondendo a 6,077 e $28,126 \mathrm{~g} \mathrm{ha}^{-1}$ de sulfentrazone, respectivamente, indicaram a capacidade de sorção desse herbicida aos constituintes do solo. A relação de sorção (RA) calculada entre o $\mathrm{I}_{50} \mathrm{em}$ areia e o do solo $\left(\mathrm{g} \mathrm{ha}^{-1}\right)$ foi de, aproximadamente, 3,6 para o Argissolo Vermelho-Amarelo. A sorção de sulfentrazone foi, conforme Grey et al. (1997), influenciada, principalmente, pelo pH e material orgânico do solo. Elevados valores de CTC também apresentaram correlação positiva com a capacidade de sorção do sulfentrazone no solo (Kerr et al., 2004). Nas condições de $\mathrm{pH}$ do solo avaliado $(6,7 \mathrm{t} 0,1)$ (Tabela 1), aproximadamente 50\% do herbicida estaria na forma aniônica, com o $\mathrm{pH}$ próximo ao pKa do herbicida $(6,56)$, o que poderia reduzir sua sorção ao solo, principalmente com baixa CTC. Entretanto, mesmo sob essas condições, a RA calculada de 3,6 vezes pode ser considerada elevada, o que favoreceria a persistência e lenta disponibilização do herbicida na solução do solo. A presença de óxidos de ferro no solo também pode ter afetado a mobilidade e capacidade de adsorção do sulfentrazone no solo, o que ocorre principalmente quando o pH deste for muito abaixo do pKa do herbicida (Alves et al., 2004), reduzindo sua eficiência em campo.

Nas avaliações de persistência do herbicida, observou-se diferença na produção de massa seca da parte aérea das plantas de sorgo somente quando estas foram cultivadas em amostras de solo da primeira coleta, correspondendo a 467 DAA. Nas demais épocas, os resíduos de sulfentrazone no solo não afetaram a produção de massa seca das plantas. Todavia, no tratamento com aplicação em 2003 e reaplicação em 2004, o sulfentrazone continuou reduzindo a produção de massa seca da parte aérea da planta indicadora até a última coleta, que ocorreu aos 640 dias da primeira aplicação, em 2003, e 197 dias após a reaplicação, em 2004 (Tabela 2). Essas plantas

Tabela 2 - Acúmulo de massa seca da parte aérea, altura e fitotoxicidade das plantas de Sorghum vulgare cultivadas em Argissolo Vermelho-Amarelo e avaliadas aos 21 dias após aplicação do herbicida, em amostras de solo $(0-10$ e $10-20 \mathrm{~cm})$ cultivado com cana-de-açúcar e tratado com sulfentrazone, para as diferentes épocas amostradas

\begin{tabular}{|c|c|c|c|c|c|c|c|c|}
\hline \multirow{4}{*}{ Aplicação } & \multicolumn{8}{|c|}{ Coletas } \\
\hline & \multicolumn{2}{|c|}{$1^{\underline{a}}$} & \multicolumn{2}{|c|}{$2^{\underline{\underline{a}}}$} & \multicolumn{2}{|c|}{$3^{-\frac{a}{4}}$} & \multicolumn{2}{|c|}{$4^{\underline{a}}$} \\
\hline & \multicolumn{8}{|c|}{ Profundidade do solo $(\mathrm{cm})$} \\
\hline & $0-10$ & $10-20$ & $0-10$ & $10-20$ & $0-10$ & $10-20$ & $0-10$ & $10-20$ \\
\hline & \multicolumn{8}{|c|}{ Massa seca da parte aérea (\% em relação à testemunha) } \\
\hline 2003 & $63,66 \mathrm{Bb}$ & $100,39 \mathrm{Aa}$ & $96,25 \mathrm{Ab}$ & $108,95 \mathrm{Aa}$ & $90,21 \mathrm{ABb}$ & $101,81 \mathrm{Aa}$ & $107,30 \mathrm{Aa}$ & $103,28 \mathrm{Aa}$ \\
\hline 2003 e 2004 & $23,35 \mathrm{Bb}$ & $71,40 \mathrm{Ba}$ & $23,83 \mathrm{Bb}$ & $99,38 \mathrm{ABa}$ & $31,45 \mathrm{ABb}$ & $111,02 \mathrm{Aa}$ & $53,94 \mathrm{Ab}$ & $99,51 \mathrm{ABa}$ \\
\hline Não aplicado & $100,00 \mathrm{Aa}$ & $100,00 \mathrm{Aa}$ & $100,00 \mathrm{Aa}$ & $100,00 \mathrm{Aa}$ & $100,00 \mathrm{Aa}$ & $100,00 \mathrm{Aa}$ & $100,00 \mathrm{Aa}$ & $100,00 \mathrm{Aa}$ \\
\hline \multirow[t]{2}{*}{$\mathrm{CV}(\%)$} & \multicolumn{8}{|c|}{12,95} \\
\hline & \multicolumn{8}{|c|}{ Altura $(\mathrm{cm})$} \\
\hline 2003 & $14,00 \mathrm{Ba}$ & $11,54 \mathrm{Ca}$ & $21,38 \mathrm{Aa}$ & $22,88 \mathrm{Aa}$ & $21,29 \mathrm{Aa}$ & $17,67 \mathrm{Ba}$ & $19,36 \mathrm{Aa}$ & $18,56 \mathrm{ABa}$ \\
\hline 2003 e 2004 & $4,08 \mathrm{Ba}$ & $9,92 \mathrm{Ba}$ & $4,83 \mathrm{Bb}$ & $18,88 \mathrm{Aa}$ & $5,92 \mathrm{Ba}$ & $17,04 \mathrm{Aa}$ & $12,75 \mathrm{Aa}$ & $16,92 \mathrm{Aa}$ \\
\hline Não aplicado & $17,75 \mathrm{Ba}$ & $13,67 \mathrm{Ba}$ & $23,54 \mathrm{Aa}$ & $21,63 \mathrm{Aa}$ & $21,58 \mathrm{ABa}$ & $18,33 \mathrm{Aa}$ & $18,92 \mathrm{Ba}$ & $17,42 \mathrm{ABa}$ \\
\hline \multirow[t]{2}{*}{$\mathrm{CV}(\%)$} & \multicolumn{8}{|c|}{10,67} \\
\hline & \multicolumn{8}{|c|}{ Fitotoxicidade (\%) } \\
\hline 2003 & $5,00 \mathrm{Aa}$ & $1,67 \mathrm{Aa}$ & $6,67 \mathrm{Aa}$ & $6,67 \mathrm{Aa}$ & $3,33 \mathrm{Aa}$ & $1,67 \mathrm{Aa}$ & $1,67 \mathrm{Aa}$ & $0,00 \mathrm{Aa}$ \\
\hline 2003 e 2004 & $83,33 \mathrm{Aa}$ & $23,33 \mathrm{Ab}$ & $91,67 \mathrm{Aa}$ & $8,33 \mathrm{Bb}$ & $81,67 \mathrm{Aa}$ & $15,00 \mathrm{ABb}$ & $33,33 \mathrm{Ba}$ & $5,00 \mathrm{Bb}$ \\
\hline Não aplicado & $0,00 \mathrm{Aa}$ & $0,00 \mathrm{Aa}$ & $0,00 \mathrm{Aa}$ & $0,00 \mathrm{Aa}$ & $0,00 \mathrm{Aa}$ & $0,00 \mathrm{Aa}$ & $0,00 \mathrm{Aa}$ & $0,00 \mathrm{Aa}$ \\
\hline CV (\%) & \multicolumn{8}{|c|}{27,78} \\
\hline
\end{tabular}

Médias seguidas por uma mesma letra maiúscula ou minúscula, na linha, não diferem entre si pelo teste de Tukey, (P>0,05). Letras maiúsculas comparam épocas de amostragem e minúsculas, as profundidades de solo em cada época amostral. 
acumularam apenas 53,94\% de massa seca em relação à testemunha que não recebeu o herbicida. Para as variáveis avaliadas, altura e fitotoxicidade, o comportamento verificado foi similar ao constatado para o percentual de acúmulo da massa seca da parte aérea, em relação à testemunha.

Quanto à movimentação do herbicida no solo, verificou-se que tanto a redução da massa seca da parte aérea e da altura quanto os indices de fitotoxicidade foram evidentes somente na profundidade de 0 a $10 \mathrm{~cm}$ do solo coletado, confirmando a alta capacidade de sorção desse herbicida ao solo PVA (Tabela 2). Sintomas da presença de resíduos de sulfentrazone foram observados na profundidade de 10 a $20 \mathrm{~cm}$, apenas para o tratamento com aplicação em 2003/2004, na primeira coleta de solo, aos 467/24 DAA, respectivamente. Embora Rossi et al. (2003) tenham avaliado em colunas de PVC a mobilidade do sulfentrazone em condições laboratoriais, os autores também constataram pequena mobilidade do herbicida em Nitossolo Vermelho (até 7,5 cm) e em Neossolo Quartzarênico (até $12,5 \mathrm{~cm})$, mesmo quando submetidos a $90 \mathrm{~mm}$ de precipitação pluvial. Já segundo Benedito et al. (1999), o sulfentrazone foi lixiviado para camadas de profundidade superior a $10 \mathrm{~cm}$ no perfil do solo, quando submetido à irrigação de 20 mm, 24 horas após a sua pulverização. Portanto, mesmo que o sulfentrazone não tenha representado risco de lixiviação para camadas inferiores do solo PVA, nas condições experimentais observadas, ressalta-se que o seu uso prolongado em uma mesma área pode modificar o seu comportamento, podendo alcançar maiores profundidades no perfil do solo.

Os resíduos (mg $\mathrm{kg}^{-1}$ por solo de sulfentrazone) estimados por bioensaio concentraram-se na camada de $0-10 \mathrm{~cm}$ de profundidade, independentemente do período amostral. Observa-se, na Figura 4, que os maiores níveis residuais $\left(0,420 \mathrm{mg} \mathrm{kg}^{-1}\right.$ de solo) ocorrem no tratamento que recebeu o herbicida em 2003 com reaplicação em 2004, sendo detectado na profundidade de 0 a $10 \mathrm{~cm} \mathrm{do}$ perfil do solo.

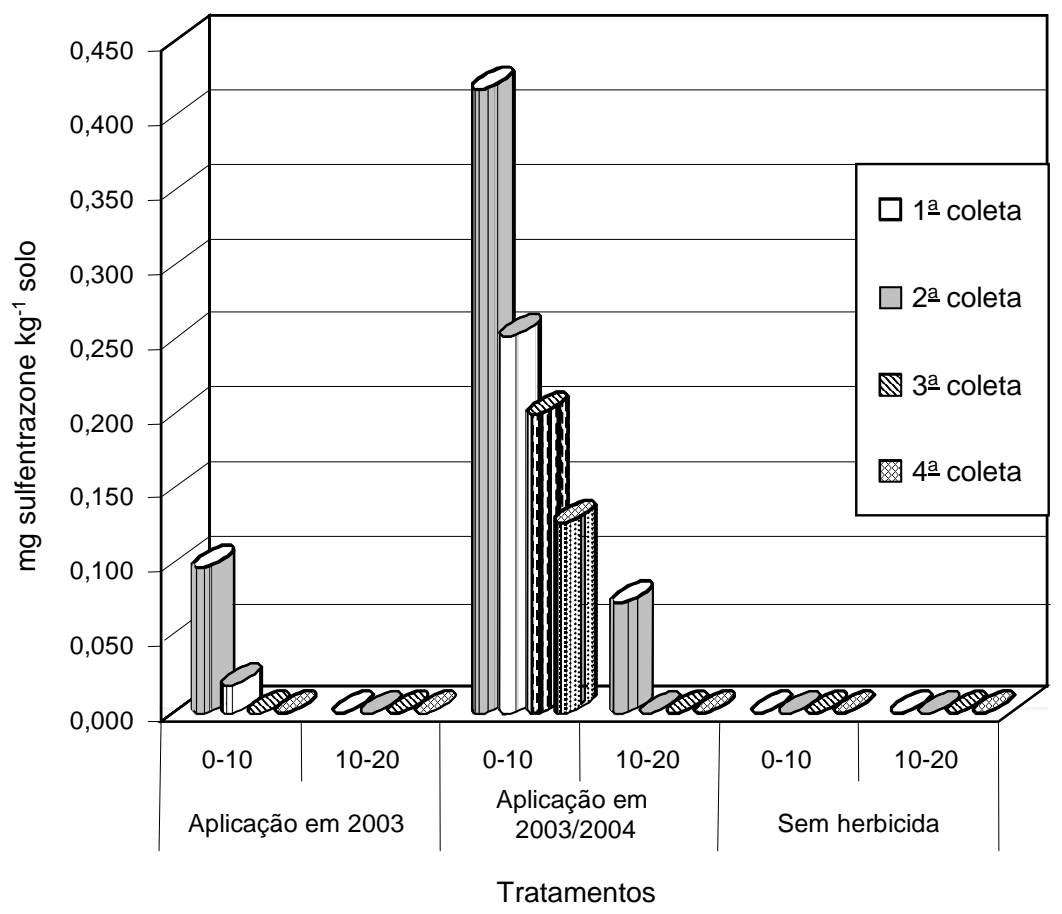

Figura 4 - Resíduos estimados de sulfentrazone por bioensaio, nas profundidades de 0-10 e 10-20 cm, em Argissolo VermelhoAmarelo submetido à pulverização desse herbicida somente em 2003, em 2003 com reaplicação em 2004 ou sem a sua aplicação (testemunha), no cultivo de cana-de-açúcar, para diferentes épocas de coleta do solo (1ª: 467/24, 2ạ: 517/74, 3ạ: 550/107 e 4a: 640/197 dias após a aplicação em 2003/2004, respectivamente). 
A elevada persistência do sulfentrazone, observada mesmo após 640/197 DAA - 2003/ $2004\left(0,128 \mathrm{mg} \mathrm{kg}^{-1}\right.$ por solo), indica o alto risco de fitotoxicidade às culturas em sucessão à cana-de-açúcar, principalmente quando esse herbicida for reaplicado em cana-soca. Estudos similares foram conduzidos por Main et al. (2004) para determinar o efeito residual carryover de sulfentrazone, aplicado em anos anteriores, sobre o cultivo do algodão. A pesquisa envolveu diferentes solos e localidades do Tennessee, Carolina do Norte, Arkansas e Oklahoma - EUA, durante os cultivos de 1998 a 2000. Os resultados revelaram reduções superiores a 30\% no rendimento do algodão em Oklahoma e de $20 \%$ nos estados de Arkansas e Carolina do Norte, correlacionandose $\left(R^{2}=0,84\right)$ com as avaliações visuais de injúrias na cultura. Esses resultados estão de acordo com a $t_{1 / 2}$ observado na literatura, podendo seu efeito residual no solo se prolongar por mais de 280 DAA.

A alta sorção e baixa dessorção garantem ao sulfentrazone longa permanência no solo, a qual é favorecida também pela sua baixa mineralização (Reddy \& Locke, 1998). A partir das avaliações de evolução de $\mathrm{C}-\mathrm{CO}_{2}$ realizadas durante 28 dias de incubação, constatou-se o efeito negativo do herbicida sobre os microrganismos do solo (Tabela 3). Nos quatro períodos de análise, o C- $\mathrm{CO}_{2}$ acumulado foi maior para o solo coletado nas parcelas sem o uso de sulfentrazone, seguido pelo tratamento com aplicação somente em 2003, com os menores valores de $\mathrm{C}-\mathrm{CO}_{2}$ observados no solo pulverizado em 2003 e 2004. Na Figura 5, observase o comportamento da evolução de $\mathrm{C}-\mathrm{CO}_{2}$, constatando-se que em todos os períodos de avaliação (07, 14, 21 e 28 dias de incubação) as médias do tratamento testemunha (sem herbicida) foram superiores às obtidas nos demais tratamentos. As menores médias verificadas para CBM e os maiores valores calculados para $\mathrm{qCO}_{2}$, nos tratamentos com o uso do sulfentrazone (Tabela 3), também comprovaram a interferência desse herbicida sobre a microbiota do solo, o que poderia afetar os processos de ciclagem e mineralização do material orgânico. Arruda et al. (2001) constataram a redução na nodulação da soja por Bradyrhizobium japonicum devido ao efeito indireto das doses do herbicida sulfentrazone nas plantas, causando inclusive efeito negativo sobre o crescimento destas.
Tabela 3 - Médias da evolução de C- $\mathrm{CO}_{2}$ acumulado, carbono da biomassa microbiana (CBM) e quociente metabólico $\left(\mathrm{qCO}_{2}\right)$ obtidos em amostras de Argissolo VermelhoAmarelo $(0-10 \mathrm{~cm})$ coletadas aos 640/197 dias após aplicação do herbicida sulfentrazone (2003/2004, respectivamente), na cultura da cana-de-açúcar, e incubadas em laboratório a $80 \%$ da capacidade de campo

\begin{tabular}{|c|c|c|c|}
\hline \multirow{2}{*}{$\begin{array}{c}\text { Avaliação } \\
\text { (dias) }\end{array}$} & \multicolumn{3}{|c|}{$\mathrm{C}-\mathrm{CO}_{2}\left(\mathrm{mg} \mathrm{kg}^{-1}\right.$ solo $)$} \\
\hline & $\begin{array}{l}\text { Aplicação em } \\
2003\end{array}$ & $\begin{array}{l}\text { Aplicação em } \\
\text { 2003/2004 }\end{array}$ & $\begin{array}{l}\text { Testemunha sem } \\
\text { herbicida }\end{array}$ \\
\hline 07 & 111,32 & 92,77 & 137,29 \\
\hline 14 & 252,33 & 222,64 & 319,12 \\
\hline 21 & 489,21 & 474,80 & 586,56 \\
\hline 28 & 685,97 & 641,30 & 737,92 \\
\hline $\mathrm{CV}(\%)$ & & 29,09 & \\
\hline \multirow{2}{*}{28} & \multicolumn{3}{|c|}{ CBM (mg kg${ }^{-1}$ de solo)* } \\
\hline & 88,68 & 76,74 & 107,36 \\
\hline $\mathrm{CV}(\%)$ & & 12,63 & \\
\hline \multirow{2}{*}{28} & \multicolumn{3}{|c|}{$\mathrm{qCO}_{2}\left(\mathrm{mg} \mathrm{mg}^{-1} \mathrm{~d}^{-1} \text { de } \mathrm{C}-\mathrm{CO}_{2} \text { do } \mathrm{CBM}\right)^{*}$} \\
\hline & 0,279 & 0,299 & 0,246 \\
\hline $\mathrm{CV}(\%)$ & \multicolumn{3}{|c|}{19,15} \\
\hline
\end{tabular}

* Valores médios obtidos a partir das análises realizadas aos 28 dias de incubação do solo.

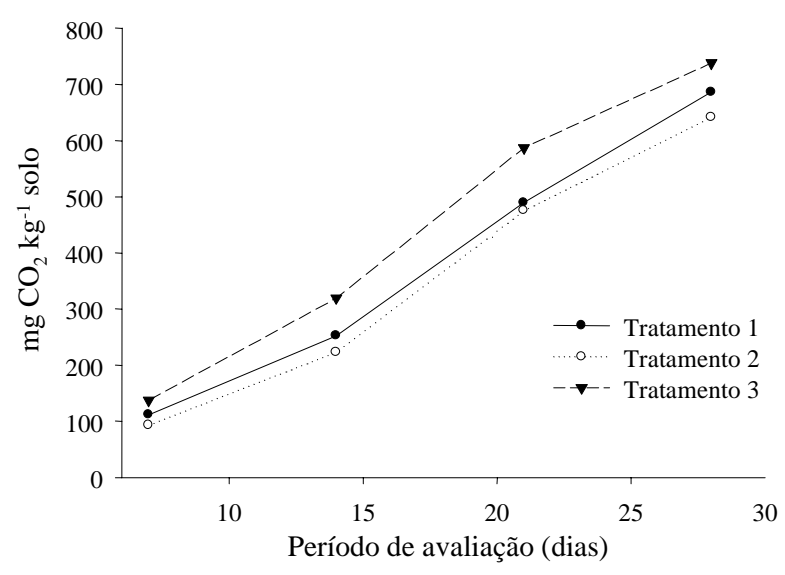

Figura 5 - Valores médios acumulados da evolução do $\mathrm{C}-\mathrm{CO}_{2}$ a partir de amostras de solo $(0-10 \mathrm{~cm})$ coletadas na última avaliação em campo, correspondente aos 640 dias após a primeira aplicação em 2003 e 197 dias da reaplicação em 2004, e incubadas em laboratório durante 28 dias. Tratamentos: 1) aplicação do herbicida somente em 2003; 2) aplicação em 2003 e 2004; 3) testemunha sem herbicida.

Ao avaliar o efeito conjunto do herbicida sobre a microbiota do solo, verifica-se a formação de dois grupamentos distintos no dendrograma (Figura 6) entre os tratamentos 


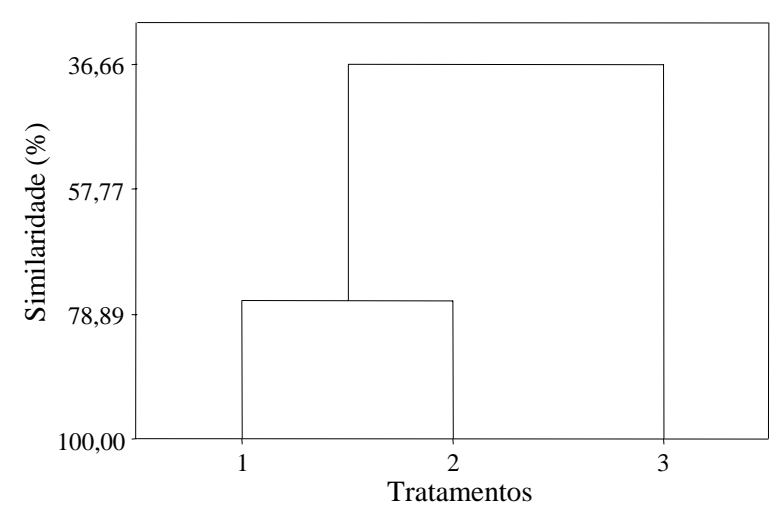

Figura 6 - Dendrograma de similaridade para a microbiota do solo Argissolo Vermelho-Amarelo (PVA), a partir das variáveis evolução de $\mathrm{C}-\mathrm{CO}_{2}$, carbono da biomassa microbiana (CBM) e quociente metabólico $\left(\mathrm{qCO}_{2}\right)$, para os tratamentos: (1) aplicação de sulfentrazone somente em 2003; (2) aplicação em 2003 e 2004; (3) testemunha sem aplicação do herbicida.

1 e 2 em relação ao tratamento 3 (testemunha sem herbicida), com percentual de similaridade entre os agrupamentos de apenas 36,6\%. A avaliação conjunta, realizada com base nos valores de evolução de $\mathrm{C}-\mathrm{CO}_{2}$, carbono da biomassa microbiana e quociente metabólico, permite constatar a interferência do sulfentrazone na atividade microbiológica do solo, necessitando-se de mais estudos para a quantificação de sua ação em longo prazo. Verifica-se que, embora conste que o mecanismo inicial de degradação do sulfentrazone seja microbiológico (FMC crop., 1995), outros estudos também comprovaram a sua ação tóxica sobre microrganismos do solo (Reddy \& Locke, 1998; Chang et al., 2001); por essa razão, esse herbicida deve ser usado com cautela. Além disso, conhecer os processos de supressão, redução da atividade e seleção microbiológica do solo por esse herbicida pode auxiliar na redução do seu efeito residual.

\section{LITERATURA CITADA}

ALVES, P. L. C.; JUNIOR, J. M.; FERRAUDO, A. S. Soil attributes and the efficiency of sulfentrazone on control of purple nutsedge (Cyperus rotundus L). Sci. Agric., v. 61, n. 3, p. 319-325, 2004.

ANDERSON, J. P.; DOMSCH, K. H. The metabolic quotient for $\mathrm{CO}_{2}\left(\mathrm{qCO}_{2}\right)$ as a specific activity parameter to asses the effects of environmental conditions, such as $\mathrm{pH}$, on the microbial biomass of forest soils. Soil Biol. Biochem., v. 25, p. 393-395, 1993.
ARRUDA, J. S.; LOPES, N. F.; BACARIN, M. A.

Nodulação e fixação do nitrogênio em soja tratada com sulfentrazone. Pesq. Agropec. Bras., v. 36, n. 2, p.325-330, 2001.

BENEDITO N. et al. Influência da cobertura morta no comportamento do herbicida sulfentrazone. Planta Daninha, v. 17 , n. 3, p. 445-458, 1999.

BLANCO, H. G. Destino, comportamento e resíduos dos herbicidas no solo. O Biológico, v. 45, p. 225-248, 1979.

CARTER, A. D. Herbicide movement in soils: principles, pathways and processes. Weed Res., v. 40, p. 113-122, 2000.

CHANG, Y. J. Impact of herbicides on the abundance structure of indigenous beta-subgroup ammonia-oxidizer communities in soil microcosms. Environ. Toxicol. Chem., v. 20, p. $2462-2468,2001$.

EUROPEAN WEED RESEARCH COUNCIL - EWRC. Cite of methods in weed research. Weed Res., v. 4, p. 88, 1964.

FILIZOLA, H. F. et al. Monitoramento e avaliação do risco de contaminação por pesticidas em água superficial e subterrânea na região de Guairá. Pesq. Agropec. Bras., v. 37 , n. 5 , p. $659-667,2002$.

FMC Corp. Technical bulletin of sulfentrazone. Philadelphia: 1995. 6 p.

GONÇALVES, A. H; SILVA, J. B; LUNKES, J. A. Controle da tiririca (Cyperus rotundus) e efeito residual sobre a cultura do feijão do herbicida imazapyr. Planta Daninha, v. 19, n. 3, p. 435-443, 2001.

GREY, T. L. et al. Behavior of sulfentrazone in ionic exchange resins, electrophoresis gels, and cation-saturated soils. Weed Sci., v. 48, n. 2, p. 239-247, 2000.

GREY, T. L. et al. Sulfentrazone adsorption and mobility as affected by soil and pH. Weed Sci., v. 45, n. 5, p. 733-738, 1997.

HINZ, C. Description of sorption data with isotherm equations. Geoderma, v. 99 p. 225-243, 2001.

HULTING, A. G. et al. Soybean (Glycine $\max$ (L.) Merr.) cultivar tolerance to sulfentrazone. Crop Protec., v. 20, p. $679-683,2001$.

ISLAM, K. R.; WEIL, R. R. Microwave irradiation of soil four routine measurement of microbial biomass carbon. Biol. Fertil. Soils, v. 27, p. 408-416, 1998.

KERR, G. W.; STAHLMAN, P. W.; DILLE J. A. Soil pH and cation exchange capacity effects sunflower tolerance to sulfentrazone. Weed Technol., v. 18, n. 2, p. 243-247, 2004. 
LAW, S. E. Agricultural electrostatic spray application: a review of significant research and development during de $2^{\text {th }}$ century. Athens: University of Georgia, 2001. p. 25-42.

MAIN, C. L. et al. Sulfentrazone persistence in southern soils: bioavailable concentration and effect on a rotational cotton crop. Weed Technol., v. 18, n. 2, p. 346-352, 2004.

POLUBESOVA, T. et al. Sulfentrazone adsorbed on micellemontmorillonite complexes for slow release in soils. $\mathbf{J}$. Agric. Food Chem., v. 51, p. 3410-3414, 2003.

REDDY, K. N.; LOCKE, M. A. Sulfentrazone sorption, desorption, and mineralization in soils from two tillage systems. Weed Sci., v. 46, n. 4, p. 494-500, 1998.

ROSSI, C. V. S.; ALVES, P. L. C. A.; MARQUES JUNIOR, J. Mobilidade do sulfentrazone em Nitossolo Vermelho e em Neossolo Quartzarênico. Planta Daninha, v. 21, n. 1, p. 111-120, 2003.

SANTOS, J. B. et al. Atividade microbiana do solo após aplicação de herbicidas em sistemas de plantio direto e convencional. Planta Daninha, v. 23, n. 4, p. 683-691, 2005.

SEEFELDT, S. S.; JENSEN, S. E.; FUERST, E. P. Loglogistic analysis of herbicide dose-response relationship. Weed Technol., v. 9, p. 218-227, 1995.

SILVA, A, A. et al. Efeito residual no solo dos herbicidas imazamox e imazethapyr para as culturas de milho e sorgo. Planta Daninha, v. 17, n. 3, p. 345-354, 1999.
STORK, P.; HANNAH, M. C. A bioassay method for formulation testing and residue studies of sulfonylureia and sulfonanylide herbicides. Weed Res., v. 36, p. 271-281, 1995.

SZMIGIELSKA, A. M.; SCHOENAU, J. J.; GREER, K. Comparasion of chemical extraction and bioassay for measurement of metsulfuron in soil. Weed Sci., v. 46, p. 487-493, 1998.

TÓTOLA, M. R.; CHAER, G. M. Microrganismos e processos microbiológicos como indicadores da qualidade dos solos. In: ALVAREZ V., V. H. et al. (Eds.). Tópicos em ciência do solo. Viçosa, MG: Sociedade Brasileira de Ciência do Solo, 2002, v. 1. p. 196-276.

VALPASSOS, M. A. R. et al. Effects of soils management systems on soil microbial activity bulk density and chemical properties. Pesq. Agropec. Bras., v. 36, n. 12, p. 1539-1545, 2001.

VANCE, E. D. et al. An extraction method for measuring soil microbial biomass C. Soil Biol. Biochem., v. 19, p. 703-707, 1987.

WEBER, B. J.; WILKERSON, G. G.; REINHARDT, C. F. Calculating pesticide sorption coefficients $(\mathrm{Kd})$ using selected soil properties. Chenosphere, v. 55, p. 157-166, 2004. 